\title{
Editorial
}

\section{The magic number}

Since the novel of Douglas Adams, The Hitchhiker's Guide to the Galaxy (1979), where the answer of a supercomputer to "the most important question", including life and the universe, is the number 42 , no one has been so attached to a single number as scientists.

This is the purpose that we often assigned to P-values, an arbitrary threshold delimiting "statistical significance". Recently, more than 800 scientists from 50 countries signed a letter requesting the withdrawal of all concepts of Statistical Significance ${ }^{1}$. The dichotomized discussion involves the terms statistically "significant" and "non-significant", which depend on whether the P-value falls below or above this "magic" number, giving the idea that the items evaluated are categorically different.

Unfortunately, the false belief that crossing the threshold of statistical significance is enough to show that a result is 'real' has led scientists and us (journal editors) to privilege such results, thereby distorting the literature. Some could argue that an arbitrary but clear limit is better than an unclear one. Additionally, if we do not use statistical significance, then what should we be doing instead? Whatever the discussion might lead to in the future, what seems relevant now is to educate ourselves about statistical misconceptions, but further, we should consider the logic, the background knowledge, and the experimental design along with $\mathrm{P}$ value to conclude.

Austral Journal of Veterinary Sciences is not considering changing how it considers the statistical analysis in evaluation of papers at this time, but we are aware of this discussion in the scientific world since the conclusions will necessarily have an impact on what is currently considered suitable for publication.

Editorial Committee Austral Journal of Veterinary Sciences

\footnotetext{
https://www.nature.com/articles/d41586-019-00857-9
} 